\title{
The evolution of the anterior capsulotomy
}

\author{
Dorota Wyględowska-Promieńska ${ }^{1,2}$, Marcin Jaworski², Kamila Kozieł², Richard Packard ${ }^{3}$ \\ 1Department of Ophthalmology, School of Medicine in Katowice, Medical University of Silesia, Katowice, Poland \\ ${ }^{2}$ Department of Ophthalmology, The University Clinical Centre Prof. Kornel Gibiński, Medical University of Silesia, Katowice, Poland \\ ${ }^{3}$ Arnott Eye Associates, Optegra Eye Hospital London, London, United Kingdom
}

Videosurgery Miniinv 2019; 14 (1): 12-18 DOI: https://doi.org/10.5114/wiitm.2019.81313

\begin{abstract}
The paper describes the development of the anterior capsulotomy from its early crude beginnings in the $18^{\text {th }}$ century to the possibility of automated surgery today via continuous curvilinear capsulorhexis (CCC). The reasons for the opening of the capsule have changed from a roughly made tear to allow access to the nucleus for its extraction, to the creation of more regular openings to allow support for intraocular lenses. With the development of continuous circular tears it was possible to be certain to contain the intraocular lens (IOL) in the capsular bag. In recent times we have the ability to achieve precision in size and location with lasers and other technologies. This means the capsulotomy can be used to hold the 1OL, which will improve the centration of the optic. This is important in premium lenses and should improve predictability of the effective lens position. All of these changes will be highlighted with appropriate illustrations.
\end{abstract}

Key words: cataract, phacoemulsification, anterior capsulotomy, continuous curvilinear capsulorhexis.

In 1747 the world of cataract surgery was changed by Jacques Daviel when instead of pushing the cataractous lens backwards into the vitreous he extracted the opaque nucleus from the eye. In order to do this he had to open the lens capsule, which he did by tearing it crudely with a cystitome.

For the next two centuries and more the techniques to open the capsule hardly altered. The invention of the intraocular lens changed things. Harold Ridley, who first placed his disc intraocular lens (IOL) in the posterior chamber, had no idea if this lens was inside or outside of the capsular bag. The lens was inherently unstable partly because of this but also due to its excessive weight when compared with a modern IOL.

Cornelius Binkhorst, one of the most important pioneers of implantology, was the first to realise that if you could fixate the IOL into the capsular bag it would be more stable and less likely to irritate ocular tissues. Accordingly, he designed an IOL which he called iridocapsular to be partly supported by the iris but mainly by the capsular bag. Binkhorst's problem was that he could not decide on the best shape for the capsular opening (Photo $1 \mathrm{~A}$ ).

Others tried to develop different ways to open the capsule, such as Albert Galand's envelope technique. Here a linear opening was made proximal to the incision and after removal of the lens nucleus, cortical clearance and IOL insertion the anterior capsule was torn open in a continuous manner after making a small scissor cut at the capsular edge.

At the beginning of the 1970s, Charles Kelman, the inventor of phacoemulsification, used a different method to gain access to the lens nucleus to emulsify it intracamerally through a small incision. He tore the capsule in a triangular manner, the so-called

\section{Address for correspondence}

Marcin Jaworski, Department of Ophthalmology, The University Clinical Centre Prof. Kornel Gibiński, Medical University of Silesia,

35 Ceglana St, 40-514 Katowice, Poland, phone: +48 3235812 00, e-mail: katedra.okulistyki@uck.katowice.pl 

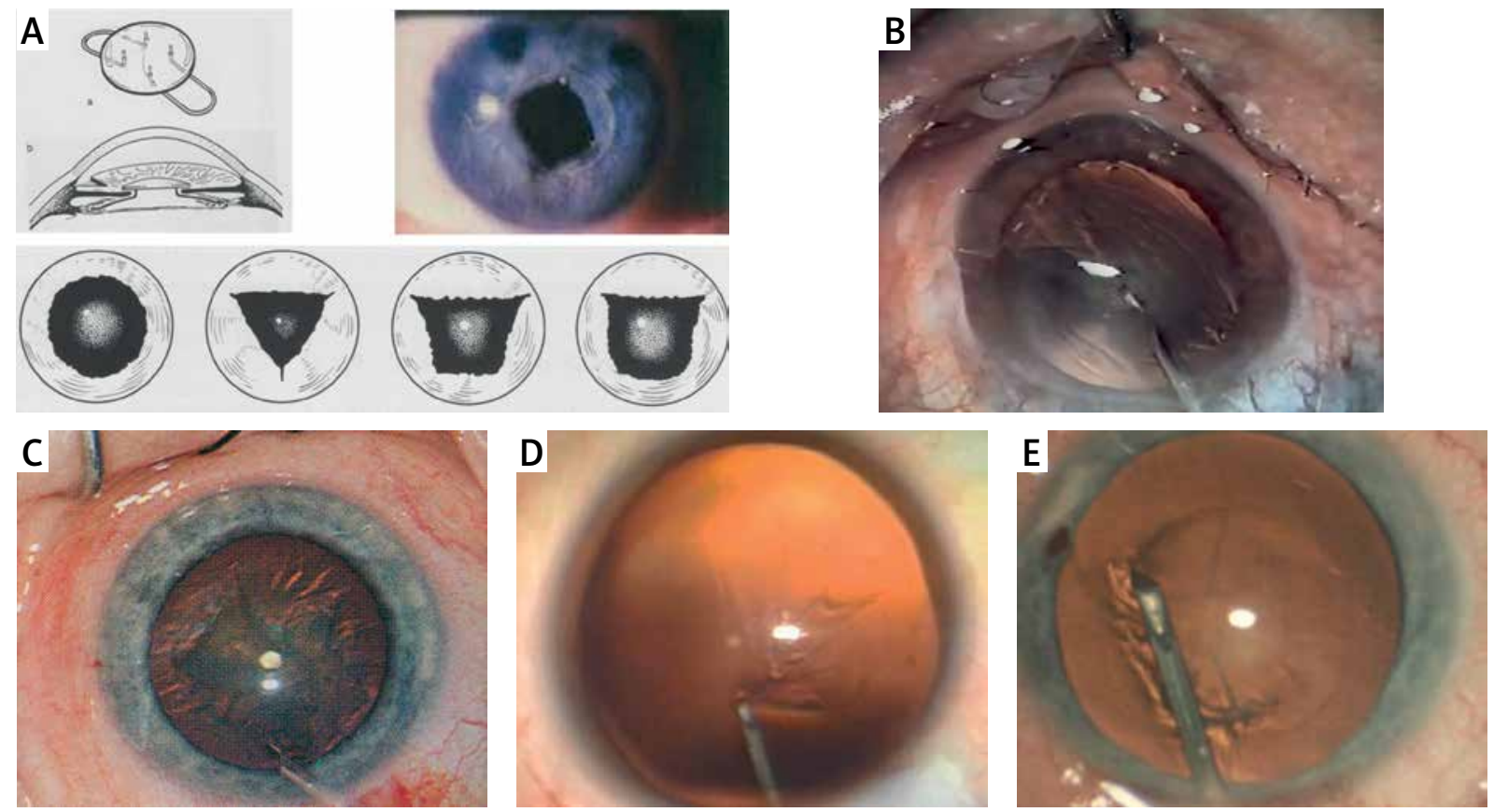

$\mathrm{F}$
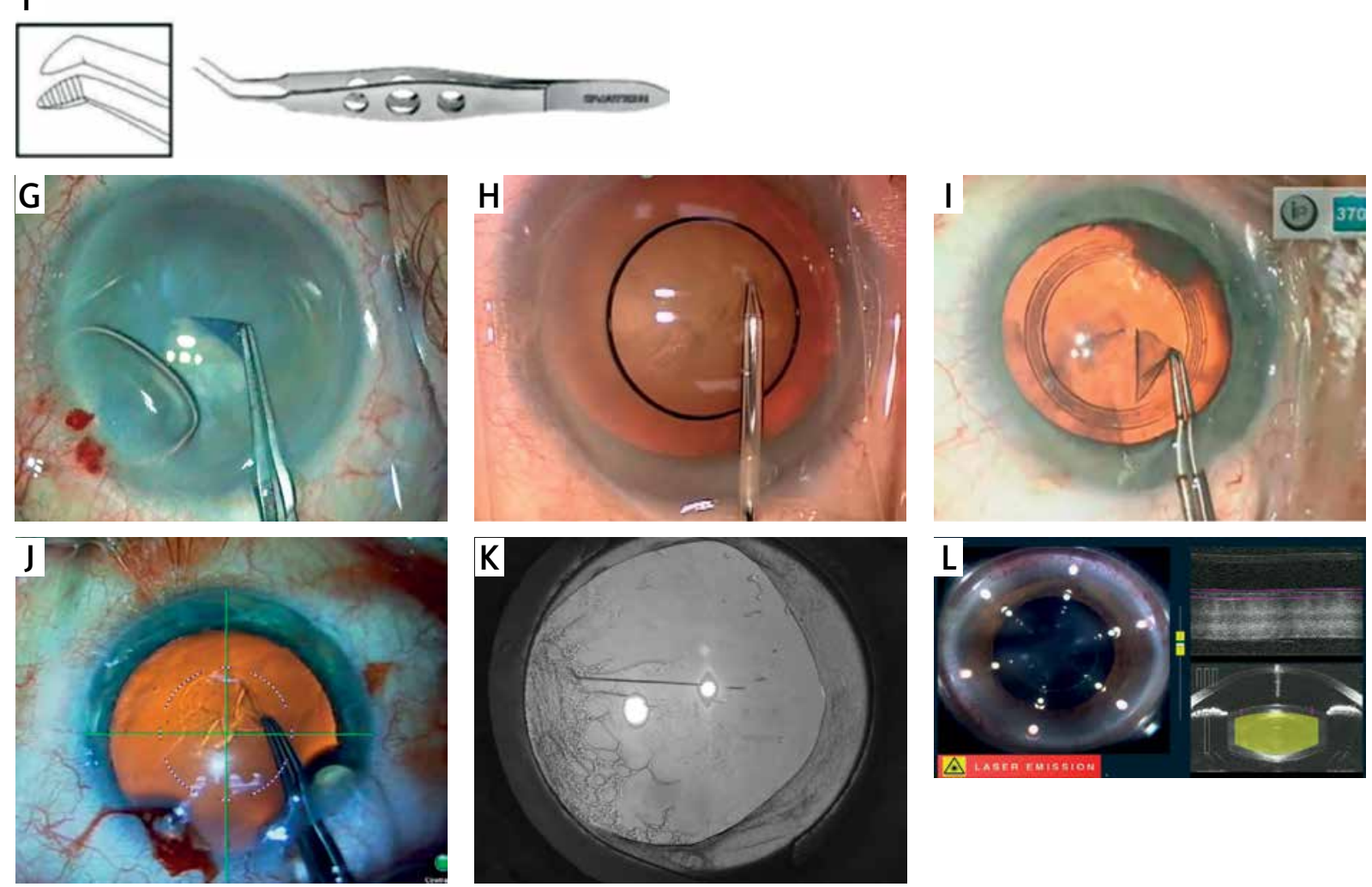

Photo 1. A - Binkhorst iridocapsular lens and capsulotomy shapes. B - Christmas tree capsulotomy. C - Can opener capsulotomy. D - Shimizu capsulotomy. E - Neuhann capsulotomy. F - Utrata forceps. G - Capsulotomy with trypan blue stained capsule. $\mathrm{H}$ - Tassignon capsulotomy ring. I - Verus capsulotomy ring. J - Digital overlay in microscope view. $\mathbf{K}$ - Asymmetric capsulotomy with posterior capsule opacification. $\mathbf{L}$ - Imaging during capsulotomy with femtosecond laser. All figures are the property of Richard Packard 
"Christmas tree" capsulotomy (Photo 1 B), and then trimmed the third side of the triangle with scissors.

When in the late 1970s Richard Kratz wanted to perform iris plane phacoemulsification and tilt the lens nucleus he devised a round capsulotomy with a serrated edge called the "can opener" (Photo $1 \mathrm{C}$ ). It was also felt that this type of capsulotomy would hold a posterior chamber IOL better.

However, none of these and other attempts to create a capsular opening was immune from tearing at the edge. The surgeon, when implanting an IOL, could also not be certain that the whole of the IOL was in the bag or that it would stay there, as the capsule contracted in the weeks and months after cataract surgery.

The first attempt to use a different modality was made in the early 1980s by the inventor of the YAG laser for posterior capsulotomy, Danielle Aron Rosa, using her laser to open the anterior capsule. Multiple punctures were made in a circular pattern. Unfortunately, unless the cataract surgery was carried out immediately the intraocular pressure would rise rapidly as the soft lens matter expanded due to hydration by aqueous and surgery became difficult. This was quickly abandoned.

The solution to remedy these problems turned out to be a continuous circular tear in the capsule. For most ophthalmologists two surgeons have been credited with pioneering the technique called continuous curvilinear capsulorhexis (CCC), but in fact the story is more complex. Thomas Neuhann in Germany and Howard Gimbel in Canada have received most recognition for this and certainly produced the first paper to appear in the peer reviewed literature [1]. However, across the world and at the same time Kimiya Shimizu was doing something very similar. Each of these three surgeons added a part of the name for this capsulotomy. Gimbel called it continuous, Shimizu added curvilinear and Neuhann coined the term capsulorhexis. Although these surgeons had a similar idea, their approaches as to how to create the CCC and the instruments they used differed.

Charles Kelman had designed an instrument called the irrigating cystitome, and this is what Howard Gimbel used. He modified the can opener technique by pulling on the capsule to create the tear but not allowing the edge to become serrated but remain continuous. He performed this under viscoelastic.

Kimiya Shimizu used a bent 30 gauge needle under viscoelastic to create a tear and then a flap of anterior capsule. He then folded this flap onto the untorn capsule and with the tip of the needle directed the tear in a curvilinear manner very much as many surgeons do today (Photo $1 \mathrm{D}$ ).

One of the authors (RP) first saw the technique that Thomas Neuhann first used for his capsulorhexis at a conference in Bordeaux in 1986. It was performed during live surgery by another German surgeon, Jurgen Greite. He had been taught by Thomas Neuhann, as they both worked in Munich. The Neuhann technique was performed with irrigation initially with a straight 26 gauge needle. This needle created a small T-shaped opening in the anterior capsule just in front of the main incision site. Then a curved needle with a bent tip was inserted into the eye and the small flaps created by the capsular incision were stroked up each side to create the CCC (Photo $1 \mathrm{E}$ ).

But were these three really the first to perform a CCC? It seems not. A surgeon in the United States called Calvin Fercho was almost certainly the first to do this. He started using a continuous tear in the late 1970s, and it is documented by a number of surgeons who watched him operate [2]. He did not present this though due to taking time out for ill health until 1986. His technique was a sort of combination of that used by Gimbel and Shimizu. He used an irrigating cystitome initially with the irrigation on but later under viscoelastic and tore the capsule with this then directed the flap to make the CCC.

Although many surgeons still use only a needle or cystitome to create the CCC, many use forceps. The first forceps specifically designed to perform this capsulorhexis were designed by Peter Utrata and appeared in 1988. Although there have been many variations on this theme, the basic design is still used widely today (Photo $1 \mathrm{~F}$ ). The reason many surgeons prefer to use forceps is the greater controllability which they offer.

One of the unintended consequences of adopting the CCC was in the way that the nucleus was removed from the eye with phacoemulsification. Tilting up the nucleus as in the Kratz technique was now much more difficult so new methods were needed. This led to "chip and flip", "divide and conquer", "phaco chop", "phaco pre-chop" and many others. The major advantages were that the edge of the capsulotomy was much stronger with less tendency to tear out. Irrigation aspiration was safer as there were not torn edges to suck into the aspiration port. The 
IOL could now be reliably placed within the capsular bag and was likely to stay there, well centred.

This new capsulotomy needed a good red reflex to be easy to perform and certainly a hypermature white cataract presented even the most experienced surgeon with a major challenge. Many would revert to a "can opener" capsular opening in these cases. A device using high frequency radio diathermy was invented by Kloti to overcome this problem. It made the capsulotomy in white cataracts much easier but the capsular edge was not as strong as a CCC.

The problem of how to deal with these mature lenses with poor red reflex was solved by using a vital stain trypan blue to stain the anterior capsule. Although Gerrit Melles is generally credited with this idea, Minas Coroneo first used this whilst working in the Australian outback operating on aboriginal patients. He holds a patent on the idea (Photo $1 \mathrm{G}$ ).

Although obtaining a complete CCC is relatively straightforward for the experienced surgeon, are there ways to make the size and circularity of the capsular opening more precise? A number of techniques have been described to do this. Marie Jose Tassignon has designed a ring which is slipped into the eye and centred on the pupil to act as a guide for the surgeon (Photo $1 \mathrm{H}$ ). Alternatively there is the Verus capsulotomy guide, a soft flat circular device which is placed on the anterior capsule and acts as a guide for the tearing of the capsule (Photo $1 \mathrm{I}$ ). Marking the cornea with a specially sized circular instrument can also aid the surgeon.

Head up displays in the surgeon's view down an operating microscope of a circle displayed on the cornea as provided in devices such as Verion (Alcon, USA) or Callisto (Zeiss, Germany) can also help to achieve greater accuracy (Photo $1 \mathrm{~J})$ ). A recent paper from Haellssler-Sinangin et al. showed that even with experienced surgeons these devices can improve the CCC [2].

Why was the CCC important?

- IOL centration and stability particularly for multifocal and toric IOLs.

- IOL effective lens position.

- Overlapping of the IOL edge with the capsule.

- Shrink wrapping of the IOL by the capsule to lessen PCO.

Emma Hollick with David Spalton's team have shown us that a poorly centred CCC without good edge coverage of the IOL leads to earlier posterior capsular opacification (Photo $1 \mathrm{~K}$ ) [3]. Little changed for another decade until the first use of the femtosecond laser for cataract surgery in 2008 by Zoltan Nagy in Hungary. This was a fundamental change in the way that the anterior capsulotomy was performed. For the first time we could make capsulotomies of a given size which were truly circular, in a given position, with little risk of tearing out during the capsulotomy, without the variables of a manual technique. The imaging devices built into the machine assisted in all of this (Photo $1 \mathrm{~L}$ ).

However, there were caveats with this new technology. A second room might be needed for the laser which interfered with the surgical flow. Both the cost of the device and the running costs are high and there is a click fee for patients. So far no study has shown advantages of femtosecond laser assisted cataract surgery (FLACS) in terms of patient outcome [4]. When asked in a twin eye study where one eye had FLACS and the other conventional surgery, patients preferred the conventional approach [5].

Early studies from Nagy and his group had shown us the accuracy of the laser capsulotomy [6], but Abell et al. demonstrated why the incidence of capsular tags and tearout during surgery was higher than in manual CCC [7]. This was due to the femtosecond laser producing a serrated edge due to its being a pulsed laser. There were also redundant laser spots due to laser spot scatter during the creation of the capsulotomy (Photo $2 \mathrm{~A}$ ), although this has to some extent been resolved by reducing the power in some studies or the vertical spot spacing in others [8] (Photo 2 B).

However, most surgeons using FLACS say that the most useful function of the laser is the way it makes the capsulotomy. Manning et al. in a multicentre case-control study compared femtosecond laser-assisted cataract surgery (FLACS) and conventional phacoemulsification cataract surgery. The researchers matched 2,814 FLACS to 4,987 conventional phacoemulsification procedures. Baseline corrected distance visual acuity (CDVA), age, number of ocular comorbidities, and surgical risk factors were taken into consideration. Citing the results: $0.7 \%$ vs. $0.4 \%$; postoperative logMAR CDVA, 0.05 $\left(6 / 6^{-3}\right)$ vs. $0.03\left(6 / 6^{-2}\right)$; worse postoperative CDVA at follow-up (by 5 letters or more), $1.0 \%$ vs. $0.4 \%$; CDVA $0.3(6 / 12)$ or better, $96.3 \%$ vs. $97.1 \%$; absolute biometry prediction error, 0.43 dioptres (D) vs. $0.40 \mathrm{D}$; within $\pm 0.5 \mathrm{D}$ of target, $72 \%$ vs. $74.3 \%$; and postoperative complications, $3.4 \%$ vs. $2.3 \%$. 

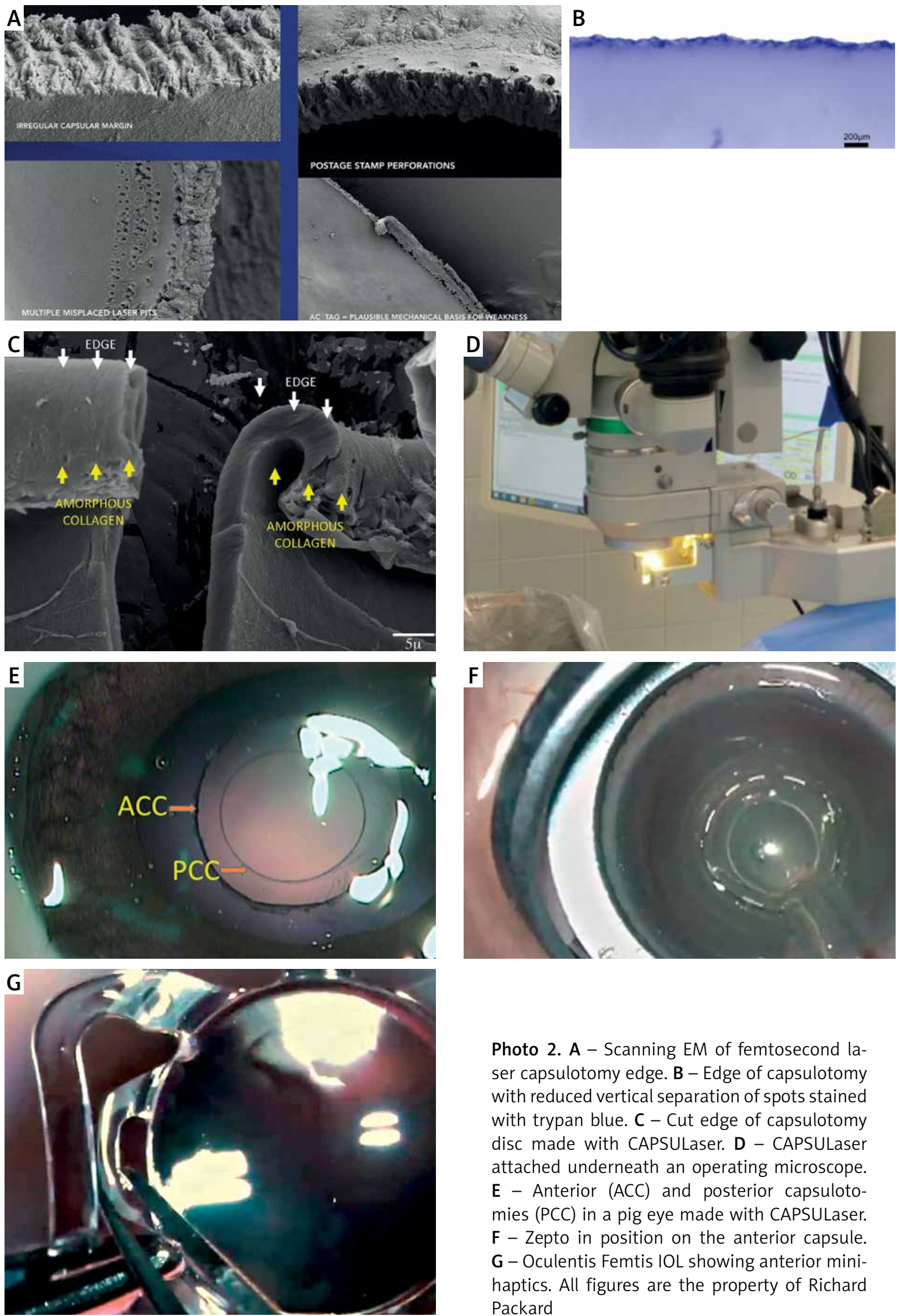

Photo 2. A - Scanning EM of femtosecond laser capsulotomy edge. B - Edge of capsulotomy with reduced vertical separation of spots stained with trypan blue. C - Cut edge of capsulotomy disc made with CAPSULaser. D - CAPSULaser attached underneath an operating microscope. E - Anterior (ACC) and posterior capsulotomies (PCC) in a pig eye made with CAPSULaser. $\mathbf{F}$ - Zepto in position on the anterior capsule. G - Oculentis Femtis IOL showing anterior minihaptics. All figures are the property of Richard Packard 
It was concluded that both FLACS and conventional phacoemulsification cataract surgery provided excellent visual outcomes. However, no evidence to support claims that FLACS is superior to conventional phaco was found. Moreover, the conventional surgery has a lower postoperative complication rate [9]. Might there be other ways to create round, consistently sized capsulotomies?

Another laser, "CAPSULaser" (Excellens, USA), has been under investigation and will soon be available commercially. It is a thermal laser which uses as its target an anterior capsule stained with an optimised solution of trypan blue. Unlike the femtosecond laser it acts in a continuous manner to create the capsulotomy. The absorption of the laser light, which is in the red/orange part of the spectrum, causes the type IV collagen in the capsule to change to amorphous collagen. The edge of the capsulotomy is rolled and smooth and in vitro paired cadaver eye tests have shown it to be more elastic than that found in manual capsulotomies and FLACS (Photo $2 \mathrm{C}$ ). Although this is a thermal laser, the time taken to create the capsulotomy is only 1 second and the overall rise in temperature in the anterior chamber is less than $0.2^{\circ} \mathrm{C}$. The beam is centred by the patient with a fixation light and focused by the surgeon using light sources built in to the device. Over 500 human eyes have been studied including most recently a CE mark study. The CE mark has now been awarded. This study showed a consistency of sizing of $99 \%$ within $50 \mu$ of $5 \mathrm{~mm}$. Circularity was similarly found. Unlike the femtosecond laser, this device is small and attaches to the underneath of an operating microscope (Photo $2 \mathrm{D}$ ) and does not interfere with the operating flow. The costs of using this device will be much lower than a femtosecond laser. It is worth noting that posterior capsulotomies have been successfully performed in vitro through IOLs and human studies will begin soon (Photo $2 \mathrm{E}$ ).

There are other thermal devices becoming available. Already on the market is a device called "Zepto". This consists of a suction ring containing a wire made of nitinol. Nitinol is a metal alloy which has memory so that when it is deformed it will return to its original shape. The device, which is single use, is pushed into the eye through the phaco wound, normally $2.5 \mathrm{~mm}$, then placed on the anterior capsule. Once it has been centred by the surgeon, suction is applied to attach the device to the capsule (Photo $2 \mathrm{~F}$ ).
Then a short electric charge is activated to cut the capsule by causing a phase transition in water molecules with a localised but small rise in temperature [10]. This device has also been used in the laboratory for a posterior capsulotomy. In a recent study from Brendan Vote's group they found that even in the latest version of the device there was a $4 \%$ tearout rate, which they attributed to tags created by the suction release mechanism [11].

Another not dissimilar approach is that provided by a metal ring also pushed in to the anterior chamber called "Aperture Rx". So far this has not been used on humans. It also acts with localised heat on the anterior capsule to create the capsulotomy.

The anterior capsulotomy has come a long way from its crude beginnings in the $18^{\text {th }}$ century. Its role has changed also from allowing access to the lens nucleus for its expression to a means of holding an IOL. In this latter role IOL manufacturers are now realising the possibility of using the perfectly sized and centred capsulotomies created by lasers to hold the $\mathrm{IOL}$ in place (Photo $2 \mathrm{G}$ ). It is to be hoped that this will improve refractive outcomes further by making the effective lens position more predictable.

\section{Acknowledgments}

All figures are the property of Richard Packard.

\section{Conflict of interest}

Richard Packard is a consultant to Excel-Lens (USA), which produces CAPSULaser. The other authors declare that there is no conflict of interest regarding the publication of this manuscript.

\section{References}

1. Gimbel H, Neuhann T. Development, advantages, and methods of the continuous circular capsulorhexis technique. J Cataract Refract Surg 1990; 16: 31-7.

2. Haeussler-Sinangin Y, Dahlhoff D, Schultz T, Dick HB. Clinical performance in continuous curvilinear capsulorhexis creation supported by a digital image guidance system. J Cataract Refract Surg 2017; 43: 348-52.

3. Hollick EJ, Spalton DJ, Meacock WR. The effect of capsulorhexis size on posterior capsular opacification: one-year results of a randomized prospective trial. Am J Ophthalmol 1999; 128: 271-9.

4. Popovic M, Campos-Muller X, Schlenker M, Ahmed I. Efficacy and safety of femtosecond laser-assisted cataract surgery compared with manual cataract surgery: a meta-analysis of 14567 eyes. Ophthalmology 2016; 123: 2113-26. 
5. Mursch-Edlmayr A, Bolz M, Luft N, et al. Intraindividual comparison between femtosecond laser-assisted and conventional cataract surgery. I Cataract Refract Surg 2017; 43: 215-22.

6. Nagy Z, Takacs A, Filkorn T, Sarayba M. Initial clinical evaluation of an intraocular femtosecond laser in cataract surgery. J Refract Surg 2009; 25: 1053-60.

7. Abell RG, Darian-Smith E, Kan JB, et al. Femtosecond laser-assisted cataract surgery versus standard phacoemulsification cataract surgery: outcomes and safety in more than 4000 cases at a single center. J Cataract Refract Surg 2015; 41: 47-52.

8. Schultz T, Joachim S, Noristani R, et al. Greater vertical spot spacingto improve femtosecond laser capsulotomy quality. J Cataract Refract Surg 2017; 43: 353-7.

9. Manning S, Barry P, Henry Y, et al. Femtosecond laser-assisted cataract surgery versus standard phacoemulsification cataract surgery: study from the European Registry of quality outcomes for cataract and refractive surgery. I Cataract Refract Surg 2016; 42: 1779-90.

10. Chang DF, Mamalis N, Werner L. Precision pulse capsulotomy: preclinical safety and performance of a new capsulotomy device. Ophthalmology 2016; 123: 255-64.

11. Hooshmand J, Abell R, Allen P, et al. Intraoperative performance and ultrastructural integrity of human capsulotomies created by the improved precision pulse capsulotomy device. J Cataract Refract Surg 2018; 44: 1333-5.

Received: 26.11.2018, accepted: 23.12.2018. 